\title{
Editorial: Special Issue on Mental Health Issues in Autism Spectrum Disorder
}

\author{
Jill C. Fodstad ${ }^{1}$
}

Published online: 22 May 2019

(C) Springer Science+Business Media, LLC, part of Springer Nature 2019

Individuals with autism spectrum disorder (ASD) are high risk for developing comorbid symptoms associated with a secondary behavioral or psychiatric disorder diagnosis. Historically, when co-occurring psychiatric symptoms occurred in individuals with ASD - regardless of cognitive status - they were attributed to ASD itself, a phenomenon referred to as diagnostic overshadowing (Simonoff et al. 2008). However, as clinical descriptions and mounting research have accumulated pointing to chronic and debilitating psychiatric symptoms over and beyond what would be expected, it cannot be assumed anymore that these difficulties arise purely due to core ASD symptoms (i.e., social communication and restricted and repetitive interests). It is rare that individuals with ASD do not experience co-occurring symptoms at some point in their lifetime (Lever and Geurts 2016). In addition to the core symptoms of ASD, comorbid psychological concerns included general psychopathologies, anxiety, depression, schizophrenia, psychosis, obsessive-compulsive disorder, tic disorders, attention deficit hyperactivity disorder, bipolar disorder, language impairment, catatonia, eating disorders, hoarding, borderline personality disorder, and oppositional defiant disorder (Mannion et al. 2014; Matson and Goldin 2013). Further, those with ASD often experience a multitude of medical, physical, or biopsychosocial conditions or phenomena that can further exacerbate underlying mental health symptoms. Approximately $70 \%$ of individuals with ASD experience at least one co-occurring concern (Dominick et al. 2007; Leyfer et al. 2006), with the high rate of comorbidity emerging in very young children (Fodstad et al. 2010) and persisting well into adulthood (Gadke et al. 2016; Lever and Geurts 2016;

Jill C. Fodstad

jfodstad@iupui.edu

1 Department of Psychiatry, IU Health Neuroscience Center, Indiana University School of Medicine, 355 W. 16th Street, Suite 4800, Indianapolis, IN 46202, USA
Lugo-Marín et al. 2019). Not surprisingly, most individuals with ASD experience even greater impairment when more than one co-occurring condition occurs.

The literature on co-occurring psychopathology in those with ASD is still growing. However, now is the time to begin to amass what we now know to help inform clinical practice and to identify gaps that should be addressed through further research. The purpose of this special issue is to assist with collating what is presently known in the area of mental health and wellness in persons with ASD. In this special issue, six high-quality reviews are presented that begin to delve into the complexities and nuances related to psychopathology, mental health, and general wellness as they relate to persons with ASD. The topics addressed here are varied but equally important and span across age, ability, and setting. The authors discuss the impact that ASD has on symptom presentation as well as provide readers with information that they can incorporate to improve diagnostic clarity and treatment outcome.

Applied behavior analysis (ABA), a summative collection of strategies based upon the law of operant conditioning, is the most effective treatment modality for individuals diagnosed with ASD (National Autism Center 2009; National Institute of Mental Health 2007). While there is strong support in the application of ABA-based procedures for those with ASD in the areas of assessment and treatment of problem behaviors and in the teaching of socially significant behaviors, there is less evidence for ABA in specifically targeting psychiatric symptoms and conditions regardless of ASD status. While ABA will continue to be used to improve functioning in core deficit areas, adaptations must be made to ensure that cooccurring and interfering conditions are addressed. Two articles in this special issue (Raches et al. in press; Ramey et al. in press) specifically address specific modifications to improve the durability of ABA interventions. Raches et al. (in press) highlight the importance of incorporating relationshipbuilding strategies through applied early childhood mental health practices $(\mathrm{I} / \mathrm{ECMH})$ with families of young children with ASD. Their review and resulting family-centered 
behavior therapy framework demonstrate how blending I/ ECMH and EIBI principles (which are complimentary yet distinct in their approach) sets the stage for improved psychiatric, behavioral, developmental, and psychosocial outcomes for the child with ASD but also their caregivers and siblings. Ramey et al. (in press) discuss how fluctuations in mood are indices of clinically significant change in the quality of life for those with ASD receiving behavioral intervention services. While incorporating an ASD-informed dependent measure of mood was found to be feasible and useful in assessing individual improvement, the authors recommended that further investigations should identify measurement methods that are not influenced or hindered by the individual with ASD's cognitive or verbal status.

Three articles (Adams et al. in press; Muskett et al. in press; Rumball in press) delve more specifically into the complexities that arise when anxiety disorders or post-traumatic stress disorder co-occurs in individuals diagnosed with ASD. Adams et al. (in press) explore the difficulties of assessing symptoms of anxiety in the school setting. The impact of ASD and co-occurring anxiety on the classroom environment, peer functioning, and in educational outcomes has been noted by researchers, clinicians, teachers, and parents (REF); however, the authors' review of 32 articles suggests that there is great variability across raters and the methods by which anxiety symptoms are characterized may not be presently appropriate. Despite teachers being in the ideal position to observe and report on co-occurring anxiety, difficulties still exist with respect to diagnostic overshadowing and use of measures standardized for non-ASD populations. More research is necessary to understand how to best characterize anxiety symptoms in children with ASD in the school setting to allow educators to identify those children who may need additional supports to lessen the impact of co-occurring symptoms and increase educational success.

Muskett et al. (in press) present a brief review on the role that sensory sensitivity has on the development, maintenance, and treatment of specific phobia in persons with ASD. Defined as a negative response to specific sensory input, sensory sensitivity is a core feature of ASD that is noted to be highly correlated with anxiety such that a bi-directional relationship arises further increasing the likelihood that specific phobias become conditioned at higher rates than in non-ASD populations. To be able to best treat specific phobias in those with ASD, acknowledging the role that sensory sensitivity has and including strategies to address core symptoms of ASD are essential. The authors present a protocol for future research to adapt evidenced-based cognitive behavior therapy (CBT) for specific phobias to best address sensory sensitivity for those with ASD, thereby increasing the likelihood that enhanced and positive outcomes occur.

In addition to increased rates of anxiety disorders, individuals with ASD are high risk for trauma exposure that may lead to subsequent PTSD or other stress-based disorders (Kerns et al. 2015). Rumball (in press) provides an overview of the current state of the field of research into PTSD in those with ASD. Across the 24 studies reviewed (most case studies), symptoms consistent with PTSD in children and adolescents were found to co-occur at a similar or greater rate compared with same-aged peers. Despite Rumball asserting that the literature base is woefully scant given the severe implications that trauma can have on a person's level of functioning level and quality of life, incorporating routine screening trauma exposure and PTSD symptoms can and should be incorporated into standard clinical practice. Furthermore, outcomes from this review suggest that trauma-informed therapies can be effective for those with ASD provided clinicians are able to make modifications to best account for individual needs.

For many individuals with ASD, accessing augmentative or complementary therapies can further improve their overall mental health and wellness. Animal-assisted interventions have been one such complimentary modality that of late has begun to demonstrate positive outcomes with respect to psychosocial functioning for those with ASD (O'Haire 2013, 2017). Tan and Simmonds (in press) present a systematic review of 18 studies specifically focusing on the application of equine-assisted activities and therapies (EAAT), a specific modality of animal-assisted interventions, as a novel intervention to target specific difficulty areas often observed in those with ASD. Findings indicate that EAAT can result in improvement in areas of functioning such as social interaction, leisure skill development, communication, self-regulation, and psychological well-being and can be a helpful adjunctive treatment for children; however, this work is still somewhat in its infancy and much work needs to be done to determine how level of IQ, ASD severity, and level of treatment intensity may mediate response to EAAT.

Each of the six articles presented in this special issue highlights the challenges and complexities of providing care to individuals with ASD who have co-occurring mental health and psychosocial needs. All of the studies could be criticized for suffering from retaining low numbers of high-quality articles as well as limitations in findings being able to generalize across a heterogeneous population. Also, the reviews presented do not capture or fully address the range of impairment, cognitive or verbal status, or lifespan issues seen in those with ASD as it relates to assessment, diagnosis, and treatment considerations. Further, while there is a specific focus on anxiety and stress-related disorders, we know that those with ASD can experience a wide range of psychiatric symptoms and conditions. Despite these shortcomings, this special issue illustrates that despite there being increased research into the area of mental health and wellness as it pertains to individuals with $\mathrm{ASD}$, there is more work to do. To be able to understand and address how co-occurring mental health symptoms impact the diverse population of those diagnosed with ASD, research 
must continue so as clinicians, caregivers, and communities can best meet the unique needs of those affected.

\section{References}

Adams, D., Young, K., \& Keen, D. (in press). Anxiety in children with autism at school: a systematic review. Review Journal of Autism and Developmental Disorders. https://doi.org/10.1007/s40489-01900172-z.

Dominick, K. C., Davis, N. O., Lainhart, J., Tager-Flusberg, H., \& Folstein, S. (2007). Atypical behaviors in children with autism and children with a history of language impairment. Research in Developmental Disabilities, 28(2), 145-162.

Fodstad, J. C., Rojahn, J., \& Matson, J. L. (2010). Emergent comorbidity in at risk children with and without autism spectrum disorder-a cross-sectional study. Journal of Developmental and Physical Disabilities, 22(4), 381-400.

Gadke, D. L., McKinney, C., \& Oliveros, A. (2016). Autism spectrum disorder symptoms and comorbidity in emerging adults. Child Psychiatry \& Human Development, 47(2), 194-201.

Kerns, C. M., Newschaffer, C. J., \& Berkowitz, S. J. (2015). Traumatic childhood events and autism spectrum disorder. Journal of Autism and Developmental Disorders, 45(11), 3475-3486.

Lever, A. G., \& Geurts, H. M. (2016). Psychiatric co-occurring symptoms and disorders in young, middle-aged, and older adults with autism spectrum disorder. Journal of Autism and Developmental Disorders, 46(6), 1916-1930.

Leyfer, O. T., Folstein, S. E., Bacalman, S., Davis, N. O., Dinh, E., Morgan, J., et al. (2006). Comorbid psychiatric disorders in children with autism: interview development and rates of disorders. Journal of Autism and Developmental Disorders, 36(7), 849-861.

Lugo-Marín, J., Magán-Maganto, M., Rivero-Santana, A., CuellarPompa, L., Alviani, M., Jenaro-Rio, C., ... \& Canal-Bedia, R. (2019). Prevalence of psychiatric disorders in adults with autism spectrum disorder: a systematic review and meta-analysis. Research in Autism Spectrum Disorders, 59, 22-33.

Mannion, A., Brahm, M., \& Leader, G. (2014). Comorbid psychopathology in autism spectrum disorder. Review Journal of Autism and Developmental Disorders, 1(2), 124-134.

Matson, J. L., \& Goldin, R. L. (2013). Comorbidity and autism: Trends, topics and future directions. Research in Autism Spectrum Disorders, 7(10), 1228-1233.
Muskett, A., Radtke, S., White, S., \& Ollendick, T. (in press). Autism spectrum disorder and specific phobia: the role of sensory sensitivity: brief review. Review Journal of Autism and Developmental Disorders. https://doi.org/10.1007/s40489-019-00159-w.

National Autism Center. (2009). Findings and conclusions of the National Standards Project: addressing the need for evidence-based practice guidelines for autism spectrum disorders. Retrieved from https:// www.umass.edu/doegrants/wp-content/uploads/2014/04/NACStandards-Report_2009_2011.pdf.

National Institute of Mental Health. (2007). Autism spectrum disorders. Bethesda, MD.

O'Haire, M. E. (2013). Animal-assisted intervention for autism spectrum disorder: a systematic literature review. Journal of Autism and Developmental Disorders, 43(7), 1606-1622.

O'Haire, M. E. (2017). Research on animal-assisted intervention and autism spectrum disorder, 2012-2015. Applied Developmental Science, 21(3), 200-216.

Raches, C., Tomlin, A. M., \& Pratt, C. (in press). Integrating applied behavior analysis and infant/early childhood mental health: Implications for early intensive intervention in autism. Review Journal of Autism and Developmental Disorders. https://doi.org/ 10.1007/s40489-018-0148-2.

Ramey, D., Healy, O., Lang, R., Gormley, L., \& Pullen, N. (in press). Mood as a dependent variable in behavioral interventions for individuals with ASD: a systematic review. Review Journal of Autism and Developmental Disorders. https://doi.org/10.1007/s40489-01900169-8.

Rumball, F. (in press). A systematic review of the assessment and treatment of posttraumatic stress disorder in individuals with autism spectrum disorders. Review Journal of Autism and Developmental Disorders. https://doi.org/10.1007/s40489-018-0133-9.

Simonoff, E., Pickles, A., Charman, T., Chandler, S., Loucas, T., \& Baird, G. (2008). Psychiatric disorders in children with autism spectrum disorders: prevalence, comorbidity, and associated factors in a population-derived sample. Journal of the American Academy of Child \& Adolescent Psychiatry, 47(8), 921-929.

Tan, V. X.-L., \& Simmonds, J. G. (in press). Equine-assisted interventions for psychosocial functioning in children and adolescents with autism spectrum disorder: a literature review. Review Journal of Autism and Developmental Disorders. https://doi.org/10.1007/s40489-0180143-7.

Publisher's Note Springer Nature remains neutral with regard to jurisdictional claims in published maps and institutional affiliations. 